\title{
ÜBER DEN BIZYKLISCHEN BIQUADRATISCHEN ZAHLKÖRPER
}

\author{
TOMIO KUBOTA
}

Unter einem bizyklischen biquadratischen Zahlkörper verstehen wir einen absolut abelschen Zahlkörper vierten Grades, der von zwei verschiedenen quadratischen Zahlkörpern zusammengesetzt wird. Wir behandeln im folgenden die Einheiten- und Idealklassengruppe dieses Körpers, indem wir die Ergebnisse von [6] und [7] weiterführen.

Es sei $K$ ein bizyklischer biquadratischer Zahlkörper mit den drei quadratischen Teilkörpern $k_{1}, k_{2}$ und $k_{3}$. Als die Struktur der Einheitengruppe vom reellen $K$ sind dann nach [7] die sieben Typen möglich. Ist nämlich $\varepsilon_{i}$ die Grundeinheit von $k_{i}(i=1,2$ und 3$)$, so besitzt $K$ als ein System der Grundeinheiten irgendeines aus den folgenden: i) $\varepsilon_{1}, \varepsilon_{2}, \varepsilon_{3}$; ii) $\sqrt{\varepsilon_{1}}, \varepsilon_{2}, \varepsilon_{3}$; iii) $\sqrt{\varepsilon_{1}}$, $\sqrt{\varepsilon_{2}}, \varepsilon_{3}$; iv) $\sqrt{\varepsilon_{1} \varepsilon_{2}}, \varepsilon_{2}, \varepsilon_{3}$; v) $\sqrt{\varepsilon_{1} \varepsilon_{2}}, \sqrt{\varepsilon_{3}}, \varepsilon_{2}$; vi) $\sqrt{\varepsilon_{1} \varepsilon_{2}}, \sqrt{\varepsilon_{2} \varepsilon_{3}}, \sqrt{\varepsilon_{3} \varepsilon_{1}}$; vii) $\sqrt{\varepsilon_{1} \varepsilon_{2} \varepsilon_{3}}$, $\varepsilon_{2}, \varepsilon_{3}$. Wir stellen in $\S 3$ fest, zusammen mit dem entsprechenden Resultate für den imaginären $K$, dass es unendlich viele $z u$ jedem genannten Typus gehörende $K$ gibt.

Nun sei $\mathfrak{a}_{i}$ ein Ideal von $k_{i}, \mathfrak{l}_{i}$ die Idealklassengruppe von $k_{i}$ und $\mathfrak{g}_{2}$ die Idealklassengruppe von $K$. Bezeichnet man dann mit $a_{1} \times a_{2} \times a_{3}$ ein Element des direkten Produktes der drei Idealgruppen von $k_{1}, k_{2}$ und $k_{3}$, so entsteht aus der Zuordnung $\mathfrak{a}_{1} \times \mathfrak{a}_{2} \times \mathfrak{a}_{3} \rightarrow \mathfrak{a}_{1} a_{2} \mathfrak{a}_{3}$ ein Homomorphismus von $\mathfrak{h}_{1} \times \mathfrak{h}_{2} \times \mathfrak{h}_{3}$ auf eine Untergruppe $\mathfrak{S}_{0}$ von $\mathfrak{S}$. Während wir in $\$ 4$ den Kern $\mathfrak{h}_{0}$ dieses Homo-

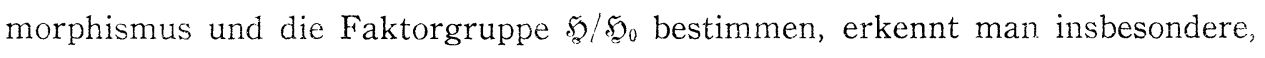
dass die beiden Gruppen $\mathfrak{h}_{0}, \mathfrak{S}_{2} / \mathfrak{S}_{0}$ Gruppen vom Typus $(2,2, \ldots)$ sind, und dass ihre Ordnungen explizite ausgedrückt werden können. Dadurch erhält man sogleich einen klassenkörpertheoretischen Beweis des von Herglotz verallgemeinerten Dirichletschen Satzes, der einmal in [6] zuerst erbracht worden ist.

Beiläufig ermöglichen unsere Resultate die Klassenzahl und ein System der Received December 7, 1955. 
Grundeinheiten von $K$ ganz leicht aus denen von $k_{i}$ zu berechnen, was in $\$ 5$ durch einige Beispiele erklärt wird.

Im folgenden wird der rationale Zahlkörper mit $P$ und die absolute Norm $N_{\Omega / P}$ bzw. die absolute Spur $S_{\Omega / P}$ mit $N_{\Omega}$ bzw. $S_{\Omega}$ bezeichnet. Überdies nehmen wir die Hassesche vereinfachte Bezeichnungsart von Gruppen an, die ein allgemeines Element einer Gruppe gleichzeitig die Gruppe selbst darstellen lässt.

\section{§ 1. Hilfssätze}

HILFSSATZ 1. Eine Einheit $\eta$ eines quadratischen Zahlkörpers $k$ hat dann und nur dann eine Darstellung $\eta=\frac{\rho^{2}}{r}(\rho \in k, r \in P)$, wenn $N_{k} \eta=1$ ist. Dabei ist notwendig $(\rho)$ ein ambiges Hauptideal von $k / P$.

Beweis. Ist $N_{k} \eta=1$, so gilt nach Hilbert $\eta=\rho^{1-s}$ mit $\rho \in k$, wo $s$ der von 1 verschiedene Automorphismus von $k / P$ ist. Also ist $\eta=\frac{\rho^{2}}{N_{k} \rho}$. Umgekehrt folgt aus $\eta=\frac{\rho^{2}}{r}$ ersichtlich $N_{k} \eta=1$. Dass dabei $(\rho)$ ein ambiges Hauptideal von $k / P$ ist, ergibt sich sofort aus $(\rho)^{2}=(r)$. Damit ist der Hilfssatz bewiesen.

Wir setzen hier die folgenden Bezeichnungen fest.

$K$ : bizyklischer biquadratischer Zahlkörper.

$k_{i}$ : quadratischer Teilkörper von $K(i=1,2$ oder 3$)$.

$\sigma_{i}:$ der Automorphismus $\neq 1$ von $K / k_{i}$.

$s_{i}: \operatorname{der}$ Automorphismus $\neq 1$ von $k_{i} / P$.

$\varepsilon_{i}$ : die Grundeinheit von $k_{i}$, wenn nur $k_{i}$ reell ist.

$\mathbf{e}_{i}$ : die Einheitengruppe von $k_{i}$.

$\mathbf{e}_{i}^{*}:$ die Gruppe aller $\eta \in \mathbf{e}_{i}$ mit $N_{k i} \eta=1$.

e: die Einheitengruppe von $K$.

Andererseits definieren wir die Zahl $\kappa(K)$ durch

$$
\kappa(K)= \begin{cases}1, & \text { wenn } K \text { reell und } N_{k_{1}} \varepsilon_{1}=N_{k_{2}} \varepsilon_{2}=N_{k_{3}} \varepsilon_{3}=-1 \text { ist. } \\ 0, & \text { sonst. }\end{cases}
$$

Falls weiter $K$ reell ist, so bezeichnen wir mit $\mathbf{e}^{*}$ die Gruppe, welche aus allen totalpositiven und totalnegativen Elementen von $\mathbf{e}_{1} \mathbf{e}_{2} \mathbf{e}_{3}$ besteht. Falls dagegen $K$ imaginär ist, so setzen wir $\mathbf{e}^{*}=\mathbf{e}_{1}^{*} \mathbf{e}_{2}^{*} \mathbf{e}_{3}^{*}$.

Aus diesen Definitionen folgt ohne weiteres der 
Hilfssatz 2. Ist $\kappa(K)=0$, so ist $\mathrm{e}^{*}=\mathbf{e}_{1}^{*} \mathbf{e}_{2}^{*} \mathbf{e}_{3}^{*}$. Ist dagegen $\kappa(K)=1$, so wird $\mathbf{e}^{*}$ aus $\mathbf{e}_{1}^{*} \mathbf{e}_{2}^{*} \mathbf{e}_{3}^{*}$ und $\varepsilon_{1} \varepsilon_{2} \varepsilon_{3}$ erzeugt. Ferner gilt immer

$$
\left(\mathbf{e}_{1} \mathbf{e}_{2} \mathbf{e}_{3}: \mathbf{e}^{*}\right)=2^{-\kappa(K)} \prod_{i}\left(\mathbf{e}_{i}: \mathbf{e}_{i}^{*}\right) .
$$

Hilfssatz 3. Es sei $K$ reell und $\kappa(K)=1$. Setzt man dann $\eta=\varepsilon_{1} \varepsilon_{2} \varepsilon_{3}$, so ist $P(\sqrt{\eta}) / P$ eine abelsche Erweiterung vom Typus $(2,2, \ldots)$. Ist ferner $k^{\prime}$ ein beliebiger Zahlkörper mit den Eigenschaften $K \cap k^{\prime}=P$ und $\sqrt{\eta} \in K^{\prime}=K k^{\prime}$, so ist $N_{K^{\prime} / k} \sqrt{\eta}=-1$.

Beweis. Da

$$
\eta, \eta^{\sigma_{1}}=\frac{\varepsilon_{1}^{2}}{\eta}, \quad \eta^{\sigma_{2}}=\frac{\varepsilon_{2}^{2}}{\eta}, \quad \eta^{\sigma_{3}}=\frac{\varepsilon_{3}^{2}}{\eta}
$$

alle Konjugierte von $\eta$ sind, hat jedes Konjugierte von $\sqrt{\eta}$ entweder die Form $\pm \sqrt{\eta}$ oder die Form $\pm \frac{\varepsilon_{i}}{\sqrt{\eta}}(i=1,2$ oder 3$)$. Andererseits ist $K$ ersichtlich in $P(\sqrt{\eta})$ enthalten, und ein durch $\sqrt{\eta} \rightarrow \pm \frac{\varepsilon_{i}}{\sqrt{\eta}}$ bestimmter Automorphismus von $P(\sqrt{\eta}) / P$ induziert in $K$ den Automorphismus $\sigma_{i}$. Also ist $P(\sqrt{\eta}) / P$ galoissch, und jeder Automorphismus $(\neq 1)$ von $P(\sqrt{\eta}) / P$ hat die Ordnung 2. Damit ist die erste Behauptung bewiesen. Betreffs der zweiten Behauptung, bedeute $\sigma_{i}^{\prime}\left(i=1,2\right.$ und 3) der Automorphismus von $K^{\prime} / k^{\prime}$, welcher $\alpha^{n_{i}^{\prime}}=\alpha^{\sigma_{i}}$ für jede $\alpha \in K$ erfüllt. Ist dann

$$
\sqrt{\eta}^{\sigma_{1}^{\prime}}=(-1)^{\nu_{1}} \frac{\varepsilon_{1}}{\sqrt{\eta}}, \quad \sqrt{\eta}^{\sigma_{2}^{\prime}}=(-1)^{\nu_{2}} \frac{\varepsilon_{2}}{\sqrt{\eta}} \quad\left(\nu_{1}, \nu_{2} \text { ganz rational }\right),
$$

so ist

$$
\sqrt{\eta}^{\sigma_{3}^{\prime}}=\sqrt{\eta}^{\sigma_{1}^{\prime} \sigma_{2}^{\prime}}=(-1)^{\nu_{1}} \cdot \frac{-1}{\varepsilon_{1}} \cdot(-1)^{\nu_{2} \sqrt{\eta}} \frac{\sqrt{\eta}}{\varepsilon_{2}}=(-1)^{v_{1}+v_{2}+1} \frac{\varepsilon_{3}}{\sqrt{\eta}} \cdot
$$

Also ist

$$
N_{K^{\prime} / k^{\prime}} \sqrt{\eta}=\sqrt{\eta}^{1+\sigma_{1^{\prime}}+\sigma_{2^{\prime}}+\sigma_{3^{\prime}}}=\sqrt{\eta} \cdot(-1)^{\nu_{1}-\frac{\varepsilon_{1}}{\sqrt{\eta}}} \cdot(-1)^{\nu_{2}-\frac{\varepsilon_{2}}{\sqrt{\eta}}}(-1)^{\nu_{1}+\nu_{2}+1} \frac{\varepsilon_{3}}{\sqrt{\eta}}=-1,
$$

w.z.b.w.

Hilfssatz 4. Eine Einheit $\eta$ von $K$ hat dann und nur dann eine Darstellung $\eta=\frac{\rho^{2}}{r}(\rho \in K, r \in P)$, wenn $\eta z u \mathrm{e}^{*}$ gehört. Insbesondere gilt $\mathrm{e}^{*} \supset \pm \mathrm{e}^{2}$.

Beweis. Es sei $\eta=\frac{\rho^{2}}{r}(\rho \in K, r \in P)$. Da dann $(\rho)^{\sigma_{i}}=(\rho)(i=1,2$ und 3$)$ 
ist, gehört $\eta_{i}=\frac{N_{K / k_{i}} \rho}{r} \mathrm{zu} \mathbf{e}_{i}$, und, falls $K$ imaginär und $k_{i}$ reell ist, so ist $N_{k_{i}} \eta_{i}=1$. Ferner gilt $\eta=\eta_{1} \eta_{2} \eta_{3}{ }^{-s_{3}}$, und für reellen $K$ ist $\eta$ der Form nach entweder totalpositiv oder totalnegativ. Daher ist $\eta \in \mathrm{e}^{*}$. Es sei umgekehrt $\eta \in \mathrm{e}^{*}$. Dann folgt aus Hilfssatz 1 , dass $\eta$ siciner eine Darstellung $\eta=\frac{\rho^{2}}{r}$ $\left(\rho \in K, r \in P\right.$ ) hat, wenn nur $\eta \in \mathrm{e}_{1}^{*} \mathrm{e}_{2}^{*} \mathrm{e}_{3}^{*}$ ist. Für die Vollendung des Beweises ist mithin hinreichend nur noch zu bestätigen, dass, im Falle von $\kappa(K)=1$, auch $\varepsilon_{1} \varepsilon_{2} \varepsilon_{3}$ sich in der Form $\frac{\rho^{2}}{r}(\rho \in K, \quad r \in P)$ darstellen lässt. Nun sei $\eta=\varepsilon_{1} \varepsilon_{2} \varepsilon_{3}, \quad N_{k_{1}} \varepsilon_{1}=N_{k_{2}} \varepsilon_{2}=N_{k_{3}} \varepsilon_{3}=-1$, und $\eta$ sei in $K$ keine Quadratzahl. Dann folgt aus Hilfssatz 3 , dass es eine $r \in P$ mit $K(\sqrt{ } \eta)=K(\sqrt{ } r)$ gibt. Da dann $\rho=\sqrt{r \eta}$ zu $K$ gehört, ist $\eta_{1}=\frac{\rho^{2}}{r}$, w.z.b.w.

Hilfssatz 5. Es sei $\rho$ eine Zahl von $K$. Gilt dann $\frac{\rho^{2}}{r}=\eta_{1} \eta_{2} \eta_{3} \eta^{2}$ mit $r \in P, \eta_{i} \in \mathrm{e}_{i}^{*}, \eta \in \mathrm{e}$, so gilt $\rho=\rho_{1} \rho_{2} \rho_{3} \eta$, wo $\rho_{i} \in k_{i}$ eine erzeugende Zahl eines ambigen Hauptideals von $k_{2} / P$ ist. Gilt umgekehrt $\rho=\rho_{1} \rho_{2} \rho_{3} \eta(\eta \in \mathbf{e})$, und ist $\left(\rho_{i}\right)$ ein ambiges Hauptideal von $k_{i} / P$, so gilt $\frac{\rho^{2}}{r}=\eta_{1} \eta_{2} \eta_{3} \eta^{2}$ mit $r \in P, \eta_{i} \in \mathrm{e}_{i}^{*}$.

Beweis. Aus $\frac{\rho^{2}}{r}=\eta_{1} \eta_{2} \eta_{3} \eta^{2}\left(\eta_{i} \in \mathrm{e}_{i}^{*}\right)$ folgt nach Hilfssatz 1 , dass $\frac{\rho^{2}}{r}=$ $\frac{\rho_{1}^{\prime 2} \rho_{3}^{\prime 2} \rho_{3}^{\prime 2}}{r^{\prime}} \eta^{2}\left(r^{\prime} \in P, \rho_{i}^{\prime} \in k_{i}\right)$ gilt, wobei $\left(\rho_{i}^{\prime}\right)$ ein ambiges Hauptideal von $k_{i} / P$ ist. $\mathrm{Da}$ dann $\frac{r}{r^{\prime}}$ in $K$ eine Quadratzahl ist, gehört $\sqrt{\frac{r}{r^{\prime}}} \mathrm{zu}$ irgendeinem $k_{l}$. Daraus folgt die erste Behauptung ${ }^{1 j}$. Ist umgekehrt $\rho=\rho_{1} \rho_{2} \rho_{3} \eta$ und $\left(\rho_{i}\right)$ ein ambiges Hauptideal von $k_{l} / P$, so ist $y_{i}=\frac{\rho_{i}^{?}}{N_{k_{\iota}} \rho_{i}}$ eine Einheit von $\mathbf{e}_{i}^{*}$. Daraus ergibt sich die zweite Behauptung.

Hilfssatz 6. Es bedeute $\rho$ eine Zahl von $K$, für die $(\rho)^{2}=(r)(r \in P)$ gilt. Ferner bedeute $\rho_{0}$ eine Zahl mit $\rho_{0}^{3} \in P$ von $K$ und $\rho_{i}$ eine $Z a h l$ von $k_{l}$, welche ein ambiges Hauptidaal $\left(\rho_{i}\right)$ von $k_{l} / P$ erzeugt. Dann entsteht aus der Zuordnung $\rho \rightarrow \rho_{r}^{\rho^{2}}$ der Isomorphismus

$$
(\rho) /\left(\rho_{0}\right) \cong \mathbf{e}^{*} / \pm \mathbf{e}^{2} \text {. }
$$

Hierbei wird die Untergruppe $\left(\rho_{1}\right)\left(\rho_{2}\right)\left(\rho_{3}\right)$ der Gruppe $\mathbf{e}_{1}^{*} \mathbf{e}_{2}^{*} \mathbf{e}_{3}^{*} \mathbf{e}^{2}$ zugeordnet.

1: Wie man leicht nach Normbildung in bezug' auf $K / k_{2}$ einsehen kann, folgt die Ambigkeit von $\left(\rho_{2}\right)$ notwendig aus $(\rho)^{2}=(\boldsymbol{r}),(\rho)=\left(\rho_{1}\right)\left(\rho_{2}\right)\left(\rho_{3}\right)$. 
Beweis. Ist $\frac{\rho^{2}}{r}= \pm \eta^{2}(\eta \in \mathrm{e})$, so ist $\left(\begin{array}{l}\rho \\ \eta\end{array}\right)^{2}= \pm r$. Also ist $(\rho)=\left(\rho_{0}\right)$ mit $\rho_{0}=\sqrt{ \pm r}$. Folglich ergeben sich alle Behauptungen aus Hilfssatz 4 und Hilfssatz 5 .

HILFSSATZ 7. Ist $\eta$ eine Einheit von $K$, so gehört $\eta^{2}$ dann und nur dann $z u \mathrm{e}_{1}^{*} \mathrm{e}_{2}^{*} \mathrm{e}_{3}^{*}$, wenn $N_{K} \eta=1$ ist. Ist insbesondere $\kappa(K)=0$, so ist $N_{k} \eta=1$ für jede Einheit $\eta$ von $K$.

Beweis. $\quad$ Da $\eta^{2}=N_{K / k_{1}} \eta \cdot N_{K / k_{2}} \eta \cdot\left(N_{K / k_{3}} \eta\right)^{-s_{3}}$ ist, folgt $\eta^{2} \in \mathbf{e}_{1}^{*} \mathbf{e}_{2}^{*} \mathbf{e}_{3}^{*}$ aus $N_{K} \eta=1$. Ist umgekehrt $\eta^{2}=\eta_{1} \eta_{2} \eta_{3}\left(\eta_{i} \in \mathrm{e}_{i}^{*}\right)$, so ist wie oben $\eta_{1} \eta_{3} \eta_{3}=N_{K / k_{1} \eta} \cdot N_{K / k_{2} \eta} \cdot\left(N_{K / k_{3}} \eta\right)^{-s_{3}}$. Nach Normbildung in bezug auf $K / k_{1}$ ist also $\eta_{1}^{2}=\left(N_{K / k_{1}} \eta\right)^{2}$, nämlich $N_{K / k_{1}} \eta= \pm \eta_{1}$, $N_{K} \eta=1$.

\section{$\S 2$. Bemerkungen über den quadratischen Zahlkörper}

Es sei $k$ ein quadratischen Zahlkörper und $\eta$ eine Einheit von $k$ mit $N_{k} \eta=1$. Dann ist nach Hilbert $\eta=\rho^{1-s}(\rho \in k)$, wo $s$ der von 1 verschiedene Automorphismus von $k / P$ ist. ${ }^{2}$ Diese Zahl $\rho$ können wir dadurch eindeutig normieren, dass $\rho$ ganz ist und durch keine rationale Primzahl teilbar ist. Die Norm $N_{k} \rho$ der so normierten Zahl $\rho$ wird im folgenden mit $\delta_{k}(\eta)$ bezeichnet.

Wir setzen hier noch die folgenden Bezeichnungen fest.

Es sei $\Omega$ ein Zahlkörper und es seien $a, b$ Zahlen von $\Omega$. Dann drücken wir mit

$$
a \overline{\bar{m}} b(\Omega)
$$

aus, dass $\frac{a}{b}$ in $\Omega$ eine $m$-te Potenz ist. Ist speziell $\Omega=P$, so schreiben wir einfach

$$
a \underset{\bar{m}}{\bar{m}} b \text {. }
$$

Dann gilt ersichtlich der

Hilfssatz 8. Es sei $k$ ein quadratischer Zahlkörper und $\eta$ eine Einheit von $k$ mit $N_{k} \eta=1$. Dann ist $\delta_{k}(\eta)$ eine für $\eta$ eindeutig bestimmte quadratfreie ganze rationale Zahl, die die Norm in bezug auf $k / P$ einer ganzen Zahl von

2) Vgl. den Beweis von Hilfssatz 1. 
$k$ ist, und die in der Diskriminante von $k$ anfgeht. ${ }^{3)}$ Ferner ist $\eta_{\overline{2}} \delta_{k}(\eta)(k)$, und fiir zwei Einheiten $\eta, \eta^{\prime}$ von $k$ gilt $\delta_{k}\left(\eta \eta^{\prime}\right) \overline{\overline{2}} \delta_{k}(\eta) \delta_{k}\left(\eta^{\prime}\right)$.

HILFSSATz 9. Es sei a eine quadratfreie positive ganze rationale Zahl und $k=P(\sqrt{\Delta})$ ein reeller quadratischer Zahlkörper. Wenn dann $N_{k} \varepsilon=1$ für die Grundeinheit $\varepsilon$ von $k$ gilt, so ist $\hat{o}_{k}(\varepsilon)$ weder mit 1 noch mit $\Delta$ identisch.

Beweis. Wäre $\delta_{k}(\varepsilon)=\Delta$, so gäbe es eine ganze Zahl $\rho$ von $k$ mit $N_{k} \rho=\Delta$, so dass $\eta=\frac{\sqrt{ } \Delta}{\rho}$ eine Einheit von $k$ mit $N_{k} \eta=-1$ sein würde, was unmöglich ist. Wäre andererseits $\delta_{k}(\varepsilon)=1$, so gehörte $z \mathrm{u} k$ eine Einheit $\eta$ mit $\varepsilon=\eta^{1-s}=\eta^{2}$ ( $s$ der Automorphismus $\neq 1$ von $k / P$ ), was wieder unmöglich ist.

HiLfSSATZ 10. $k$ sei ein reeller quadratischer Zahlkörper mit der Grundeinheit $\varepsilon$, und $\varepsilon^{*}$ bedeute $\varepsilon$ odder $\varepsilon^{2}$, je nachdem $N_{k} \varepsilon=1$ oder -1 ist. Ferner seien

$$
\begin{array}{lll}
x, x_{1}, x_{2} \text { Primzahlen } \equiv 1 & (\text { mod. } 4), \\
y, y_{1}, y_{2} \text { Primzahlen } \equiv-1 & (\text { mod. } 4) .
\end{array}
$$

Setzt mann dann $\delta=\delta_{k}\left(\varepsilon^{*}\right)$, so erhalten wir die folgenden Resultate:

i) $\delta=1$ (k) für $k=P(\sqrt{2})$, für $P(\sqrt{x})$ und für $P\left(\sqrt{x_{1} x_{2}}\right)$ mit $\left(\frac{x_{1}}{x_{2}}\right)=-1$.

ii) $\delta \overline{\overline{2}} 2$ (k) für $k=P(\sqrt{y})$ und für $P(\sqrt{2 y})$.

iii) $\delta \overline{\overline{2}} y_{1}(k)$ für $k=P\left(\sqrt{y_{1} y_{2}}\right)$.

iv) $\delta=x$ für $k=P(\sqrt{x y}) \operatorname{mit}\left(\frac{y}{x}\right)=1,\left(\frac{2}{x}\right)=-1$.

v) $\delta \overline{\overline{2}} 2 x$ (k) für $k=P(\sqrt{x y})$ mit $\left(\frac{y}{x}\right)=\left(\frac{2}{x}\right)=-1$.

vi) $\delta=y_{1} y_{2}$ für $k=P\left(\sqrt{x y_{1} y_{2}}\right)$ mit $\left(\frac{y_{1}}{x}\right)=\left(\frac{y_{2}}{x}\right)=-1$.

Beweis. Bekanntlich ist $N_{k} \varepsilon=-1$ im Falle i), und in allen anderen Fällen ist $N_{k} \varepsilon=1$. Sogar gilt im Falle iv)

$$
\left(\frac{2, x y}{x}\right)=\left(\frac{y, x y}{2}\right)=\left(\frac{2 x, x y}{x}\right)=\left(\frac{2 y, x y}{x}\right)=\left(\frac{x y, x y}{2}\right)=\left(\frac{2 x y, x y}{x}\right)=-1,
$$

im Falle v)

$$
\left(\frac{2, x y}{x}\right)=\left(\frac{x, x y}{x}\right)=\left(\frac{y, x y}{x}\right)=\left(\begin{array}{c}
x y, x y \\
2
\end{array}\right)=\left(\frac{2 x y, x y}{x}\right)=-1
$$

3) Vgl. Hilfssatz 1. 
und im Falle vi)

$$
\begin{aligned}
\left(\frac{x, x y_{1} y_{2}}{y_{1}}\right) & =\left(\begin{array}{c}
y_{1}, x y_{1} y_{2} \\
x
\end{array}\right)=\left(\begin{array}{c}
y_{2}, x y_{1} y_{2} \\
x
\end{array}\right) \\
& =\left(\frac{x y_{1}, x y_{1} y_{2}}{x}\right)=\left(\frac{x y_{2}, x y_{1} y_{2}}{x}\right)=\left(\frac{x y_{1} y_{2}, x y_{1} y_{2}}{y_{1}}\right)=-1 .
\end{aligned}
$$

Aus diesen und aus den in Hilfssatz 8 und Hilfssatz 9 ausgesprochenen Eigenschaften von $\delta$ folgen nun alle Behauptungen.

Es sei $\eta$ eine Einheit mit $N_{k} \gamma_{l}=1$ eines quadratischen Zahlkörpers $k$. Dann wird $\delta_{k}(\eta)$ als der quadratfreie Kern von $N_{k}(\eta+1)$ leicht aus $\eta$ berechnet. Unterstehend ist die Tafel von $\delta=\delta_{k}(\varepsilon)$ ( $\varepsilon$ die Grundeinheit von $k$ ) für alle $k=P(\sqrt{\Delta})$ mit $N_{k} \varepsilon=1,0<\Delta<100$.

\begin{tabular}{c|rrrrrrrrrrr}
\hline$\Delta$ & 3, & 6, & 7, & 11, & 14, & 15, & 19, & 21, & 22, & 23, & 30 \\
\hline$\delta$ & 6, & 3, & 2, & 22, & 2, & 10, & 38, & 7, & 11, & 2, & 6 \\
\hline$\Delta$ & 31, & 33, & 34, & 35, & 38, & 39, & 42, & 43, & 46, & 47, & 51 \\
\hline$\delta$ & 2, & 3, & 2, & 14, & 19, & 13, & 7, & 86, & 2, & 2, & 102 \\
\hline$\Delta$ & 55, & 57, & 59, & 62, & 66, & 67, & 69, & 70, & 71, & 77, & 78 \\
\hline$\delta$ & 5, & 19, & 118, & 2, & 33, & 134, & 3, & 14, & 2, & 11, & 3 \\
\hline$\Delta$ & 79, & 83, & 86, & 87, & 91, & 93, & 94, & 95 & & \\
\hline$\delta$ & 2, & 166, & 43, & 58, & 14, & 31, & 2, & 5 & & \\
\hline
\end{tabular}

\section{§3. Einheiten des bizyklischen biquadratischen Zahlkröpers.}

HILFSSATZ 11. Es sei $K$ ein bizyklischer biquadratischer Zahlkörper und $\eta_{i}$ eine Einheit in $\mathbf{e}_{i}^{*}(i=1,2$ oder 3$)$. Dann gehört $\eta=\eta_{1} \eta_{2} \eta_{3}$ dann und nur dann $z u \mathrm{e}^{2}$, wenn

$$
\delta_{k_{1}}\left(\eta_{1}\right) \delta_{k_{2}}\left(\eta_{2}\right) \delta_{k_{3}}\left(\eta_{3}\right)=1 \quad(K)
$$

ist, und $r_{i}$ gehört dann und nur dann $z u \mathrm{e}_{1}^{2} \mathrm{e}_{2}^{2} \mathrm{e}_{3}^{2}$, wenn zudem

$$
\delta_{k_{i}}\left(\eta_{i}\right) \overline{\overline{2}} \pm 1 \quad\left(k_{i}\right) \quad \text { für jedes } i
$$

gilt.

Beweis. Nach Hilfssatz 8 ist $\delta_{k_{1}}\left(\eta_{1}\right) \delta_{k_{2}}\left(\eta_{2}\right) \delta_{k_{3}}\left(\eta_{3}\right)=1(K)$ gleichbedeutend mit $\gamma_{l} \in \mathbf{e}^{2}$. Ist nun $\eta=\eta_{1} \eta_{2} \eta_{3} \in \mathbf{e}_{1}^{2} \mathbf{e}_{2}^{2} \mathbf{e}_{2}^{2}$, so ergibt sich nach Normbildung in bezug auf $K / k_{i}$, dass $\eta_{i} \in=\mathbf{e}_{i}^{2}$ und folglich $\delta_{k_{i}}\left(\eta_{i}\right)= \pm 1\left(k_{i}\right)$ ist. Nimmt man 
umgekehrt $\delta_{k_{1}}\left(\eta_{1}\right) \delta_{k_{2}}\left(\eta_{2}\right) \delta_{k_{3}}\left(\eta_{3}\right)=1(K)$ und $\delta_{k_{i}}\left(\eta_{i}\right) \overline{\overline{2}} \pm 1\left(k_{i}\right)$ an, so ist nach Hilfssatz $8 \eta_{i} \in \pm \mathbf{e}_{i}^{2}$ und daher $\eta_{1}=\eta_{1} \eta_{2} \eta_{3} \in \pm \mathbf{e}_{1}^{2} \mathbf{e}_{2}^{2} \mathbf{e}_{3}^{2}$. Da nach der Annahme $\sqrt{\eta} \in K$ ist, muss es notwendig $\eta \in \mathbf{e}_{1}^{2} \mathbf{e}_{2}^{2} \mathbf{e}_{3}^{2}$ sein, w.z.b.w.

Es sei wieder $\eta_{i} \in \mathbf{e}_{i}^{*} \quad(i=1,2$ und 3$)$. Dann bildet die Gesamtheit aller Beziehungen $\delta_{k_{1}}\left(\eta_{1}\right)^{m_{1}} \delta_{k_{2}}\left(\eta_{2}\right)^{m_{2}} \delta_{k_{3}}\left(\eta_{3}\right)^{m_{3}} \frac{\bar{T}_{2}}{1}(K)$ ( $m$ ganz rational) eine Gruppe, wenn man die Beziehung $\delta_{k_{1}}\left(\eta_{1}\right)^{m_{1}+m_{1}^{\prime}} \delta_{k_{2}}\left(\eta_{2}\right)^{m_{2}+m_{2}^{\prime}} \delta_{k_{3}}\left(\eta_{3}\right)^{m_{3}+m_{3}^{\prime}} \overline{\overline{2}} 1 \quad(K)$ als das Produkt zweier Beziehungen $\delta_{k_{1}}\left(\eta_{1}\right)^{m_{1}} \delta_{k_{2}}\left(\eta_{2}\right)^{m_{2}} \delta_{k_{3}}\left(\eta_{3}\right)^{m_{3}} \overline{\overline{2}} 1(K)$ und $\delta_{k_{1}}\left(\eta_{1}\right)^{m_{1}^{\prime}} \delta_{k_{2}}\left(\eta_{2}\right)^{m_{2}^{\prime}} \delta_{k_{3}}\left(\eta_{3}\right)^{m_{3}^{\prime}} \overline{\overline{2}} 1(K)$ ansieht. Diese Gruppe wird im folgenden mit $\mathbf{D}\left(\eta_{1}, \eta_{2}, \eta_{3}\right)$ bezeichnet. Die Zahl $\delta_{k_{i}}\left(\eta_{i}\right)^{m_{i}}$ (i=1,2 und 3) heisst "trivial," wenn $\delta_{k_{i}}\left(\eta_{i}\right)^{m_{i}} \overline{\overline{2}} \pm 1\left(k_{i}\right)$ ist, und die Beziehung $\delta_{k_{1}}\left(\eta_{1}\right)^{m_{1}} \delta_{k_{2}}\left(\eta_{2}\right)^{m_{2}} \delta_{k_{3}}\left(\eta_{3}\right)^{m_{3}} \overline{\overline{2}} 1(K)$ selbst heisst "trivial," wenn jede $\delta_{k_{i}}\left(\eta_{i}\right)^{m_{i}}$ "trivial" ist. Die Gesamtheit aller "trivialer" Beziehungen bildet dann eine Untergruppe von $\mathbf{D}\left(\eta_{1}, \eta_{2}, \eta_{3}\right)$, die im folgenden mit $D_{0}\left(\eta_{1}, \eta_{2}, \eta_{3}\right)$ bezeichnet wird.

SATZ 1.") Es sei $K$ ein reeller bizyklischer biquadratischer Zahlkörper mit den drei quadratischen Teilkörpern $k_{1}, k_{2}$ und $k_{3}$. Ist dann $\varepsilon_{i}$ die Grundeinheit von $k_{i}$, so besitzt $K$ als ein System der Grundeinheiten irgendeines aus den folgenden:

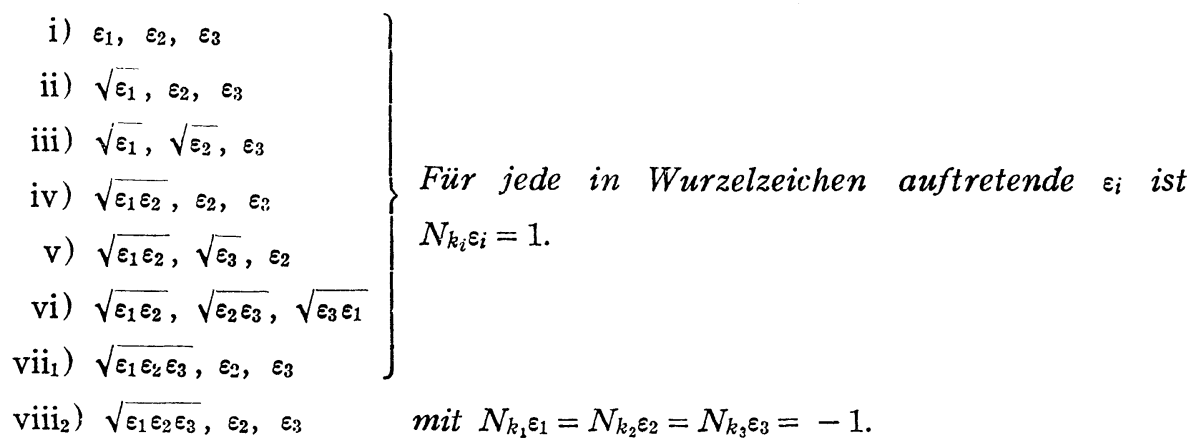

Es gibt überdies unendlich viele zu jedem Typus gehörende $K$.

Beweis. Es sei $k_{i}=P\left(\sqrt{\Delta_{i}}\right)$ mit einer quadratfreien ganzen rationalen Zahl $\Delta_{i}(i=1,2$ oder 3$)$, $\varepsilon_{i}^{*}$ bedeute $\varepsilon_{i}$ oder $\varepsilon_{i}^{2}$, je nachdem $N_{k_{i}} \varepsilon_{i}=1$ oder -1 ist, und es sei $\delta_{i}=\delta_{k_{i}}\left(\varepsilon_{i}^{*}\right)$. Ist dann erstens $\kappa(K)=0$, so folgt aus Hilfssatz 2, Hilfssatz 4 und Hilfssatz 11, dass die Zuordnung

$$
\pm \sqrt{\varepsilon_{1}^{* m_{1}} \varepsilon_{2}^{* m_{2}} \varepsilon_{3}^{* m_{3}}} \longrightarrow \delta_{1}^{m_{1}} \delta_{2}^{m_{2}} \delta_{3}^{m_{3}} \overline{\overline{2}} 1
$$

4) Vgl. [7], Satz 11 . 
einen Isomorphismus

$$
\mathbf{e} / \mathbf{e}_{1} \mathbf{e}_{2} \mathbf{e}_{3} \cong \mathbf{D}\left(\varepsilon_{1}^{*}, \varepsilon_{2}^{*}, \varepsilon_{3}^{*}\right) / \mathbf{D}_{0}\left(\varepsilon_{1}^{*}, \varepsilon_{2}^{*}, \varepsilon_{3}^{*}\right)
$$

liefert. In diesem Falle ist aber das gleichzeitige Bestehen der nicht "trivialen" Beziehungen

$$
\delta_{1} \overline{\overline{2}} 1(K), \quad \delta_{2} \overline{\overline{2}} 1(K), \quad \delta_{3} \overline{\overline{2}} 1(K)
$$

unmöglich. Wäre nämlich dies der Fall, so käme, nach Hilfssatz 8 und Hilfssatz $9,\left[2, \Delta_{i}\right] \mid\left[2, \Delta_{j}\right]$ für jedes $i, j(i, j=1,2$ oder 3$)$ vor, wo durch die eckige Klammer das kleinste gemeinsame Vielfache gemeint wird. Folglich ergäbe sich $\Delta_{i} \mid 2$ für jedes $i$, was für rellen $K$ unmöglich ist. ${ }^{5)}$ Als das Vertretersystem von $\mathbf{D}\left(\varepsilon_{1}^{*}, \varepsilon_{2}^{*}, \varepsilon_{3}^{*}\right) / \mathbf{D}_{0}\left(\varepsilon_{1}^{*}, \varepsilon_{2}^{*}, \varepsilon_{3}^{*}\right)$ wird also irgendeines aus den folgenden angenommen. ${ }^{6)}$

i) keine nicht-"triviale" Beziehung.

ii) $\delta_{1} \overline{\overline{2}} 1(K)$

iii) $\delta_{1} \overline{\overline{2}} 1(K), \delta_{2} \overline{\overline{2}} 1(K)$

iv) $\delta_{1} \delta_{2} \overline{\overline{2}} 1(K)$

v) $\delta_{1} \delta_{2} \overline{\overline{2}} 1(K), \delta_{3} \overline{\overline{2}} 1(K)$

In jeder Beziehung ist jedes auftre-

vi) $\delta_{1} \delta_{2} \overline{\overline{2}} 1(K), \delta_{2} \delta_{3} \overline{\overline{2}} 1(K)$

vii $\left.{ }_{1}\right) \delta_{1} \delta_{2} \delta_{3} \overline{\overline{2}} 1(K)$ tende $\delta_{i}$ nicht "trivial."

Der im Satz ausgesprochene Typus der Einheiten von $K$ wird dann eindeutig durch die entsprechende Beziehung zwischen $\delta_{i}$ bestimmt. Dass es unendlich viele zu jedem Typus gehörende $K$ gibt, erkennt man sogleich folgenderweise, indem man Hilfssatz 10 gebraucht.

$$
\begin{aligned}
& \text { i) } \Delta_{1}=x_{1} y, \quad \Delta_{2}=x_{2} y, \quad \Delta_{3}=x_{1} x_{2} \\
& \delta_{1}=x_{1}, \quad \delta_{2} \overline{\overline{2}} 2 x_{2}\left(k_{2}\right), \quad \delta_{3} \overline{\overline{2}} 1\left(k_{3}\right), \\
& \text { wo }\left(\frac{y}{x_{1}}\right)=1,\left(\frac{2}{x_{1}}\right)=\left(\frac{y}{x_{2}}\right)=\left(\frac{2}{x_{2}}\right)=\left(\frac{x_{1}}{x_{2}}\right)=-1 \text {. } \\
& \text { ii) } \Delta_{1}=x y, \quad \Delta_{2}=y, \quad \Delta_{3}=x \\
& \delta_{1}=x, \quad \delta_{2} \overline{\overline{2}} 2\left(k_{2}\right), \quad \delta_{3} \overline{\overline{2}} 1\left(k_{3}\right), \\
& \text { wo }\left(\frac{y}{x}\right)=1,\left(\frac{2}{x}\right)=-1 \text {. }
\end{aligned}
$$

5) Vgl. [7], S. 397.

6) Zwei Beziehungen, die voneinander nur durch eine Permutation der Indizes $i$ verschieden sind, sind hierbei als gleich anzusehen. 


$$
\begin{aligned}
& \text { iii ) } \Delta_{1}=y, \quad \Delta_{2}=2 y, \quad \Delta_{3}=2 \\
& \delta_{1} \overline{\overline{2}} 2\left(k_{1}\right), \quad \delta_{2} \overline{\overline{2}} 2\left(k_{2}\right), \quad \delta_{3} \overline{\overline{2}} 1\left(k_{3}\right) \\
& \text { iv) } A_{1}=x y, \quad \Delta_{2}=y, \quad \Delta_{3}=x \\
& \delta_{1} \overline{2} 2 x\left(k_{1}\right), \quad \delta_{2} \overline{\overline{2}} 2\left(k_{2}\right), \quad \delta_{3} \overline{\overline{2}} 1\left(k_{s}\right), \\
& \text { wo }\left(\frac{y}{x}\right)=\left(\frac{2}{x}\right)=-1 \text {. } \\
& \text { v) } \Delta_{1}=y_{1}, \quad \Delta_{2}=y_{2}, \quad \Delta_{3}=y_{1} y_{2} \\
& \delta_{1} \overline{\overline{2}} 2\left(k_{1}\right), \quad \delta_{2} \overline{\overline{2}} 2\left(k_{2}\right), \quad \delta_{3} \overline{\overline{2}} y_{1}\left(k_{3}\right) \\
& \text { vi) } \Delta_{1}=y_{1} y_{2}, \quad \Delta_{2}=y_{2} y_{3}, \quad \Delta_{3}=y_{3} y_{1} \\
& \delta_{1} \overline{\overline{2}} y_{1}\left(k_{1}\right), \quad \delta_{2} \overline{2} y_{2}\left(k_{2}\right), \quad \delta_{3} \overline{2} y_{3}\left(k_{3}\right) \\
& \text { vii } \left._{1}\right) \Delta_{1}=x y_{1} \nu_{2}, \quad \Delta_{2}=y_{1}, \quad \Delta_{3}=x y_{2} \\
& \delta_{1}=y_{1} y_{2}, \quad \delta_{2}=2\left(k_{2}\right), \quad \delta_{3}=2 x \quad\left(k_{3}\right), \\
& \text { wo }\left(\frac{y_{1}}{x}\right)=\left(\frac{y_{2}}{x}\right)=\left(\frac{2}{x}\right)=-1 \text {. }
\end{aligned}
$$

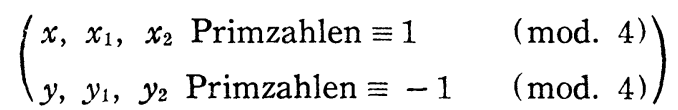

Ist zweitens $\kappa(K)=1$, so folgt aus Hilfssatz 2 und Hilfssatz 4, dass entweder $\varepsilon_{1}, \varepsilon_{2}$, $\varepsilon_{3}$ oder $\sqrt{\varepsilon_{1} \varepsilon_{2} \varepsilon_{3}}, \varepsilon_{2}, \varepsilon_{3}$ ein System der Grundeinheiten von $K$ ist. Nach Hilfssatz 6 gehört nun $\sqrt{\varepsilon_{1} \varepsilon_{2} \varepsilon_{3}}$ dann und nur dann $z \mathrm{u} K$, wenn es keine Zahl $\rho \in K$ gibt, die zwar $(\rho)^{2}=(r)(r \in P)$, aber nimmer $(\rho)=\left(\rho_{1}\right)\left(\rho_{2}\right)\left(\rho_{3}\right)\left(\rho_{i} \in k\right)$ erfüllt. Es sei $\Delta_{1}=x_{1}, \Delta_{2}=x_{2}, \Delta_{3}=x_{1} x_{2}$ mit $\left(\frac{x_{1}}{x_{2}}\right)=-1\left(x_{1}, x_{2}\right.$ Primzahlen $\equiv 1$ (mod. 4)). Dann ist bekanntlich $\kappa(K)=1$ und es gibt keine $\rho$ mit den fraglichen Eigenschaften. Daraus ergibt sich die Existenz unendlich vieler zum Typus vii ${ }_{2}$ ) gehörender Körper $K$. Der Satz ist somit vollständig bewiesen.

SATz 2. ${ }^{7)}$ Es sei $K$ ein imaginärer bizyklischer biquadratischer Zahlkörper mit dem reellen quadratischen Teilkörper $k_{1}$ und mit den imaginären quadratischen Teilkörpern $k_{2}$ und $k_{3}$. Bezeichnet man dann mit $\varepsilon_{1}$ die Grundeinheit von $k_{1}$, so ist entweder $\varepsilon_{1}$ oder $\sqrt{\zeta} \sqrt{\Sigma_{1}}$ eine Grundeinheit von $K$, wo $\zeta$ eine Einheitsuurzel mit der Eigenschaft $\sqrt{\zeta} \notin K$ von $K$ ist. Es gibt überdies unendlich viele zu jedem Typus gehörende $K$.

Beweis. Es sei $k_{i}=P\left(\sqrt{\Delta_{i}}\right), \delta_{1}=\delta_{k_{1}}\left(\varepsilon_{1}^{*}\right)$, wie beim Beweis von Satz 1 , und $\zeta_{i}(i=2$ oder 3$)$ bedeute eine erzeugende Einheitswurzel von $\mathbf{e}_{i}^{*}$. Setzt man

¿ Vgl. [7], Satz 12 . 
dann $\delta_{2}=\delta_{k_{2}}\left(\zeta_{2}\right), \delta_{3}=\delta_{k_{3}}\left(\zeta_{3}\right)$, so liefert, nach Hilfssatz 2, Hilfssatz 4 und Hilfssatz 11, die Zuordnung

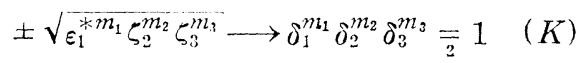

einen Isomorphismus

$$
\mathbf{e} / \mathbf{e}_{1} \mathbf{e}_{2} \mathbf{e}_{3} \cong \mathbf{D}\left(\varepsilon_{1}^{*}, \zeta_{2}, \zeta_{3}\right) / \mathbf{D}_{0}\left(\varepsilon_{1}^{*}, \zeta_{2}, \zeta_{3}\right),
$$

und $\pm \sqrt{\varepsilon_{1}^{* m_{1}} \zeta_{2}^{m_{2}} \zeta_{3}^{m_{3}}}$ ist dann und nur dann von der Form $\zeta^{\prime} \eta^{\prime}\left(\zeta^{\prime}\right.$ Einheitswurzel von $K, \eta_{1}^{\prime} \in \mathbf{e}_{1}$ ), wenn $\delta_{1}^{m_{1}}$ "trivial" ist. Ferner ist in diesem Falle das Bestehen der nicht."trivialen" Beziehung $\delta_{1}=1(K)$ unmöglich. Denn wäre dies der Fall, so hätte $K$ notwendig reell $z u$ sein. Als die nicht."trivial" $\delta_{1}$ entha1tenden Beziehungen zwischen $\delta_{i}$ sind daher nur folgende zwei möglich."

i) keine nicht-"triviale" Beziehung.

ii) $\delta_{1} \delta_{2}=1(K)$ ( $\delta_{1}$ nicht-"trivial").

Vom Typus i) sind nach Hilfssatz 10 die Körper $K$ mit

$$
\Delta_{1}=x,(x \text { Primzahl } \equiv 1(\bmod .4)),
$$

und vom Typus ii) sind die Körper mit

$$
\Delta_{1}=y_{1} y_{2}, \quad \Delta_{2}=-y_{1}, \quad \Delta_{3}=-y_{2} \quad(\sqrt{-1} \notin K)
$$

oder mit

$$
\begin{aligned}
& \Delta_{1}=y, \Delta_{2}=-1, \Delta_{3}=-y \quad(\sqrt{-1 \in K}) \\
& \left(y, y_{1}, y_{2} \text { Primzahlen } \equiv-1 \quad(\bmod .4)\right) .
\end{aligned}
$$

Damit ist der Satz bewiesen.

Gelegendlich fügen wir hier noch den folgenden Satz zu.

SATz 3. Es gibt unendlich viele reelle bizyklische biquadratische Zahlkörper $K$ mit der Eigenschaft $N_{K} \eta=1$ für jede Einheit $\eta$ von $K$, und es gibt auch unendlich viele $K$ mit der Eigenschaft $N_{K} \eta=-1$ fïr passende Einheit $\eta$ von $K$.

Beweis. Für $K$ mit $\kappa(K)=0$ ist nach Hilfssatz $7 N_{K} \mathbf{e}=1$. Für $K$ mit den Eigenschaften $\kappa(K)=1$ und $\sqrt{\varepsilon_{1} \varepsilon_{2} \varepsilon_{3}} \in K$ ist nach Hilfssatz $3 N_{K} \cdot \varepsilon_{1} \varepsilon_{2} \varepsilon_{3}=-1$. Dadurch erhält man die Behauptungen, wenn man Satz 1 berücksichtigt. 
§ 4. Idealklassengruppe des bizyklischen biquadratischen Zahlkörpers.

Wir setzen hier noch einmal die folgenden Bezeichnungen fest. ${ }^{\mathrm{s}}$

$p$ : rationale Primzahl

$e(p)$ : die Verzweigungsordnung in $K$ von $p$.

Nun heben wir vorläufig eine in Klassenkörper- und Normenresttheorie wohlbekannte Tatsache als Hilfssatz hervor.

Hilfssatz 12. Es sei $S$ der grösste über $P$ abelsche Teilkörper des absoluten Klassenkörpers von $K$. Dann ist $S / P$ vom Typus $(2,2, \ldots)$ und

$$
(S: P)= \begin{cases}\frac{1}{2} \prod_{p} e(p), & \text { wenn } K \text { reell und }-1 \text { kein Normenrest } \\ & \text { von } K / P \text { mod. irgendeine } p \text { ist. } \\ \prod_{p} e(p), & \text { sonst. }\end{cases}
$$

Hilfssatz 13. Es sei $\mathfrak{g}_{0}$ die Gruppe derjenigen Idealklassen, die durch Ideale von der Form $a_{1} a_{2} a_{3}$ vertreten werden, wo $a_{i}$ ein Ideal von $k_{i}$ bedeutet $\left(i=1,2\right.$ oder 3). Dann ist der Klassenkörper über Sू $_{0}$ der grösste über $P$ abelsche Teilkörper $S$ des absoluten Klassenkörpers von $K$.

Beweis. Der Klassenkörper über $\mathfrak{S}_{0}$ sei $S^{\prime}$. Dann gilt $\mathfrak{a}^{1-\sigma_{1}}=N_{K / k_{2}} \mathfrak{a}$ - $\left(N_{K / k_{3}} a\right)^{-s_{3}}$ für jedes Ideal a von $K$. Daher ist $S^{\prime}$ über $k_{1}$, also symmetrisch auch über $k_{2}$ und $k_{3}$, abelsch. Ist nun $\sigma^{\prime}$ ein Automorphismus von $S^{\prime} / P$, so ist, für irgendeines $i, \sigma^{\prime}=\left(\frac{S^{\prime} / k_{i}}{\mathfrak{a}_{i}}\right)$, wo $\mathfrak{a}_{i}$ ein Ideal von $k_{i}$ ist. Daraus folgt

$$
\sigma^{\prime 2}=\left(\frac{S^{\prime} / k_{i}}{a_{i}}\right)^{2}=\left(\frac{S^{\prime} / K}{\mathfrak{a}_{i}}\right)=1,
$$

so dass $S^{\prime} / P$ eine abelsche Erweiterung vom Typus $(2,2, \ldots)$ ist. Also ist $S \supset S^{\prime}$. Da aber nach Hilfssatz $12 S / P$ vom Typus $(2,2, \ldots)$ ist, ist notwendig

$$
\left(\frac{S / K}{\mathfrak{a}_{i}}\right)=\left(\frac{S / k_{i}}{\mathfrak{a}_{i}}\right)^{2}=1
$$

für jedes Ideal $a_{i}$ von jedem $k_{i}$, was $S \subset S^{\prime}$ ergibt.

Hilfssatz 14. Damit es ein Hauptideal $(\alpha)$ mit $N_{K} \alpha<0$ ( $\left.\alpha \in K\right)$ von $K$

8) Die bisherigen Festsetzungen bleiben wirklich. 
gibt, welches sich in der Form $(\alpha)=a_{1} a_{2} a_{3}$ mit Idealen $a_{i}$ von $k_{i}$ darstellen lässt, ist notwendig und hinreichend, dass -1 Normenrest von $K / P$ mod. jede $p$ ist.

Beweis. Ist $(\alpha)=a_{1} a_{2} a_{3}, N_{K} \alpha<0$, so ist ersichtlich $N_{K} \alpha=-a^{2}$ mit $a \in P$. Daraus folgt, dass -1 Normenrest von $k_{i} / P(i=1,2$ und 3$)$ und daher von $K / P$ mod. jede $p$ ist. Ist umgekehrt dies der Fall, so gibt es $\beta_{1} \in k_{1}, \beta_{2} \in k_{2}$ mit $N_{k_{1}} \beta_{1}=N_{k_{2}} \beta_{2}=-1 . \quad \mathrm{Da}$ dann $N_{K / k_{3}} \frac{\beta_{1}}{\beta_{2}}=1$ ist, ist nach Hilbert $\frac{\beta_{1}}{\beta_{2}}=\alpha^{1-\sigma_{3}}$ mit $\alpha \in K^{9)} \quad$ Also folgt aus $\alpha^{1-\sigma_{3}}=N_{K / k_{1}} \alpha \cdot\left(N_{K / k_{2}} \alpha\right)^{-s_{2}}$, dass $\frac{N_{K / k_{1}} \alpha}{\beta_{1}}=\frac{\left(N_{K / k_{2}} \alpha\right)^{s_{2}}}{\beta_{2}}$ $\in P$ ist, und dass daher $N_{K} \alpha=-a^{2}$ mit $a \in P$ gilt. Mithin ist, für ein Primideal $p$ ersten Grades von $K$, die Summe der Exponenten von $(\alpha)$ für die Konjugierten von $\mathfrak{p}$ notwendig gerade, so dass, wegen $p^{2}=N_{K / k_{1}} p \cdot N_{K / k_{2}} p \cdot\left(N_{K / k_{3}} p\right)^{-s_{3}}$, das Ideal $(\alpha)$ sich in der Form $(\alpha)=a_{1} a_{2} a_{3}$ mit Idealen $a_{i}$ von $k_{i}$ darstellen lässt, w.z.b.w.

HILFSSATZ 15. Es sei $a_{i}$ ein Ideal von $k_{i}$ und es sei $a_{1} a_{2} a_{3}=(\alpha)(\alpha \in K)$ ein Hauptideal in $K$. Dann gehört jedes $\mathfrak{a}_{i}$ notwendig zu einer ambigen Idealklasse von $k_{i} / P$. Wenn $K$ reell und $\kappa(K)=0$ ist, so hängt das Vorzeichen von $N_{K} \alpha$ nicht von der speziellen Auswahl des Erzeugenden $\alpha$ ab. Falls dabei $N_{K} \alpha>0$ ist, so gibt es für jedes $i$ eine Zahl $\rho_{i} \in k_{i}$ mit

$$
\mathfrak{a}_{i}^{1-s_{i}}=\left(\rho_{i}\right), \quad N_{k_{i}} \rho_{i}=1 \text {. }
$$

Falls aber $N_{k} \alpha<0$ ist, so gibt es solch eine $p_{i}$ nur für $i$ mit $N_{k_{i}} \epsilon_{i}=-1$. Wenn dagegen entweder $K$ imaginär ist, oder $\kappa(K)=1$ ist, so kann jedes $a_{i}^{1-s_{i}}$ von einer Zahl $\rho_{i} \in k_{i}$ mit $N_{k_{i}} \rho_{i}=1$ erzeugt werden.

Beweis. Alle Behauptungen sind ohne weiteres klar nach Hilfssatz 7 und nach

$$
a_{i}^{1-s_{i}}=\frac{\left(N_{K / k_{2}} \alpha\right)}{N_{k_{1}} a_{1} \cdot N_{k_{2}} a_{2} \cdot N_{k_{3}} a_{3}} .
$$

Hilfssatz 16. $\mathfrak{r}_{i}$ bedeute ein ambiges Ideal von $k_{i}$ und $\rho$ eine Zahl von $K$ mit $(\rho)^{2}=(r), r \in P$. Dann ist

$$
\left(\mathfrak{r}_{1} \mathfrak{r}_{2} \mathfrak{r}_{3}:(\rho)\right)= \begin{cases}2^{t-5}\left(\mathbf{e}: \mathbf{e}^{*}\right), & K \text { reell, } \\ 2^{t-3}\left(\mathbf{e}: \mathbf{e}^{*}\right), & \text { für } \quad \text { imaginär },\end{cases}
$$

9) Vgl. [2], S. 449. 
wobei $t$ die Anzahl der in $K / P$ sich verzweigenden Primzahlen ist.

Beweis. Es ist ersichtlich $\mathfrak{r}_{1} \mathfrak{r}_{2} \mathfrak{r}_{3} \supset(\rho)$ und

$$
\left(\mathfrak{r}_{1} \mathfrak{r}_{2} \mathfrak{r}_{3}:(1)\right)=2^{t} \text {. }
$$

Nun bedeute $\rho_{0}$ eine Zahl mit $\rho_{0}^{2} \in P$ von $K$. Dann ist

$$
\left(\left(\rho_{0}\right):(1)\right)= \begin{cases}2, & \text { ween } K \ni \sqrt{-1} \text { ist. } \\ 2^{2}, & \text { sonst. }\end{cases}
$$

Ferner ist nach Hilfssatz 6

$$
\left((\rho):\left(\rho_{0}\right)\right)=\left(\mathbf{e}^{*}: \pm \mathbf{e}^{2}\right) .
$$

Andererseits ist

$$
\begin{aligned}
& \left(\mathbf{e}: \mathbf{e}^{*}\right)\left(\mathbf{e}^{*}: \pm \mathbf{e}^{2}\right)=\left(\mathbf{e}: \pm \mathbf{e}^{2}\right) \\
& =\left\{\begin{array}{l}
2^{3}, \text { wenn } K \text { reell ist. } \\
2^{2}, \text { wenn } K \ni \sqrt{-1} \text { ist. } \\
2, \text { sonst. }
\end{array}\right.
\end{aligned}
$$

Daraus folgt die Behauptung, denn es ist

$$
\begin{aligned}
\left(\mathfrak{r}_{1} \mathfrak{r}_{2} \mathfrak{r}_{3}:(\rho)\right) & =\frac{\left(\mathfrak{r}_{1} \mathfrak{r}_{2} \mathfrak{r}_{3}:(1)\right)}{\left((\rho):\left(\rho_{0}\right)\right)\left(\left(\rho_{0}\right):(1)\right)} \\
& =\frac{2^{t}\left(\mathbf{e}: \mathbf{e}^{*}\right)}{\left(\left(\rho_{0}\right):(1)\right)\left(\mathbf{e}: \pm \mathbf{e}^{2}\right)} .
\end{aligned}
$$

SATz 4. Es sei $K$ ein bizyklischer biquadratischer Zahlkörper mit den drei quadratischen Teilkörpern $k_{1}, k_{2}$ und $k_{3}$. \$ $\oiint_{\text {bzw. }} \mathfrak{h}_{i}$ sei die Idealklassengruppe von $K$ bzw. $k_{i}(i=1,2$ oder 3$)$, und e bzw. $\mathbf{e}_{i}$ sei die Einheitengruppe von $K$ bzw. $k_{i} . \quad$ Es bedeuie $\mathfrak{h}_{i, 0}$ die Gruppe der ambigen Idealklassen von $k_{i} / P$, und es bedeute $\mathfrak{h}_{i}^{\prime}$, o die Gruppe derjenigen Idealklassen von $k_{i}$, welche ambige Ideale von $k_{i} / P$ enthalten. Man bezeichne mit $e(p)$ die Verzweigungsordnung in $K / P$ einer rationalen Primzahl $p$, und man bezeichne mit $\$_{0}$ bzw. $\mathfrak{H}_{0}$ das Bild bzw. der Kern des Homomorphismus von $\mathfrak{h}_{1} \times \mathfrak{h}_{2} \times \mathfrak{h}_{3}$ in $\mathfrak{S}_{\text {, }}$, der aus der natürlichen Zuordnung $a_{1} \times a_{2} \times a_{3} \rightarrow a_{1} a_{2} a_{3}$ entsteht, wo $a_{1} \times a_{2} \times a_{3}$ ein Element des direkten Produktes der drei Idealgruppen von $k_{1}, k_{2}$ und $k_{3}$ bedeutet. Schliesslich setze man

$$
\mathfrak{h}_{0}^{\prime}=\mathfrak{h}_{0} \cap \mathfrak{h}_{1,0}^{\prime} \times \mathfrak{h}_{2}^{\prime}, 0 \times \mathfrak{h}_{3,0}^{\prime}
$$


Dann sind $\mathfrak{5} / \mathfrak{y}_{0}, \mathfrak{h}_{0}$ Gruppen vom Typus $(2,2, \ldots)$, und es ist:

$$
\begin{aligned}
& \left(\mathfrak{S}_{\mathfrak{g}}: \mathfrak{S}_{0}\right)=\left\{\begin{aligned}
\frac{1}{2^{3}} \prod_{p} e(p), & \text { wenn } K \text { reell und }-1 \text { kein Normenrest } \\
& \text { von } K / P \text { mod. irgendeine rationale Prim- } \\
& \text { zahl } p \text { ist. } \\
\frac{1}{2^{2}} \prod_{p} e(p), & \text { sonst. }
\end{aligned}\right. \\
& \left(\mathfrak{h}_{0}: \mathfrak{h}_{0}^{\prime}\right)=\left\{\begin{array}{l}
2, \text { wenn } K \text { reell und }-1 \text { Normenrest von } K / P \text { mod. } \\
\quad \text { jede rationale Primzahl } p \text { ist, und wenn zudem } \\
\text { mindestens ein } k_{i} \text { keine Einheit } \eta_{i} \text { mit } N_{k_{i}} \eta_{i}=-1 \\
\text { enthält. } \\
\text { 1. sonst. }
\end{array}\right.
\end{aligned}
$$

(3) $\quad\left(\mathfrak{G}_{0}^{\prime}: 1\right)^{10)}=\left\{\begin{array}{cl}\frac{\prod_{p} e(p)}{\left(\mathbf{e}: \mathbf{e}_{1} \mathbf{e}_{2} \mathbf{e}_{3}\right)}, & \text { wenn } K \text { reell ist, und wenn zudem } \\ & \text { jeder } k_{i} \text { eine Einheit } \eta_{i} \text { mit } N_{k_{i}} y_{i}=-1 \\ \prod_{p} e(p) & \text { enthält. } \\ \frac{p}{2\left(\mathbf{e}: \mathbf{e}_{1} \mathbf{e}_{2} \mathbf{e}_{3}\right)}, & \text { sonst. }\end{array}\right.$

Beweis. Dass $\mathfrak{5} / \mathfrak{S}_{0}$ und $\mathfrak{h}_{0}$ vom Typus $(2,2, \ldots)$ sind, folgt, zusammen mit (1), unmittelbar aus Hilfssatz 12, Hilfssatz 13 und Hilfssatz 15, und (2) folgt aus Hilfssatz 14 und Hilfssatz 15. Nun sei $t$ bzw. $t_{i}$ die Anzahl der Primzahlen, die in der Diskriminante von $K / P$ bzw. $k_{i} / P$ aufgehen. Dann ist bekanntlich

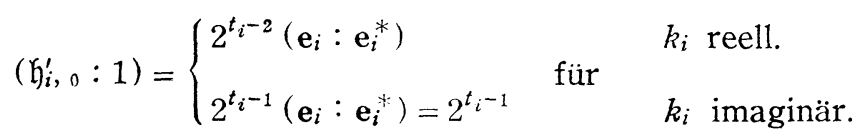

Also ist nach Hilfssatz 16

$$
\begin{aligned}
\left(\mathfrak{G}_{0}^{\prime}: 1\right) & =\frac{\prod_{i}\left(\mathfrak{G}_{i, 0}^{\prime}: 1\right)}{\left(\mathfrak{r}_{1} \mathfrak{r}_{2} \mathfrak{r}_{3}:(\rho)\right)}=\frac{2^{\Sigma_{i} t_{i}-t-1} \prod_{i}\left(\mathbf{e}_{i}: \mathbf{e}_{i}^{*}\right)}{\left(\mathbf{e}: \mathbf{e}^{*}\right)} \\
& =\frac{\prod_{p} e(p) \cdot \prod_{i}\left(\mathbf{e}_{i}: \mathbf{e}_{i}^{*}\right)}{2\left(\mathbf{e}: \mathbf{e}^{*}\right)},
\end{aligned}
$$

wo $\mathfrak{r}_{i}, \rho$ die gleichen Bedeutungen wie bei Hilfssatz 16 haben. Nun folgt aus Hilfssatz 2

10. Hierbei bedeutet 1 das Einselement der Idealklassengruppe. 


$$
\left(\mathbf{e}: \mathbf{e}^{*}\right)=2^{-\kappa(K)}\left(\mathbf{e}: \mathbf{e}_{1} \mathbf{e}_{2} \mathbf{e}_{3}\right) \prod_{i}\left(\mathbf{e}_{i}: \mathbf{e}_{i}^{*}\right)
$$

Daher ist

$$
\left(\mathfrak{h}_{0}^{\prime}: 1\right)=\frac{\prod_{p} e(p)}{2^{1-\kappa(K)}\left(\mathbf{e}: \mathbf{e}_{1} \mathbf{e}_{2} \mathbf{e}_{3}\right)},
$$

was nichts anderes als (3) ist.

SATz 5. Es sei $K$ ein bizyklischer biquadratischer Zahlkörper mit den drei quadratischen Teilkörpern $k_{1}, k_{2}$ und $k_{3}$, e bzw. $\mathbf{e}_{i}(i=1,2$ oder 3$)$ die Einheitengruppe von $K$ bzw. $k_{i}$ und $h$ bzw. $h_{i}$ die Klassenzahl von $K b z w . k_{i}$. Setzt man dann $Q=\left(\mathbf{e}: \mathbf{e}_{1} \mathbf{e}_{2} \mathbf{e}_{3}\right)$, so gilt

$$
h=\left\{\begin{array}{lll}
\frac{1}{2^{2}} Q h_{1} h_{2} h_{3} & K \text { reell. } \\
\frac{1}{2} Q h_{1} h_{2} h_{3} & \text { für } & K \text { imaginär. }
\end{array}\right.
$$

Beweis. Mit Bezeichnungen von Satz 4 ist

$$
h=\frac{\left(\mathscr{2}: \mathscr{S}_{0}\right)}{\left(\mathfrak{h}_{0}: 1\right)} h_{1} h_{2} h_{3}
$$

Man setze jetzt

$$
\begin{aligned}
& \iota=\left\{\begin{array}{l}
1, \text { wenn } K \text { reell ist. } \\
0, \text { sonst. }
\end{array}\right. \\
& \kappa=\kappa(K), \\
& \lambda=\left\{\begin{array}{l}
1, \text { wenn } K \text { reell und }-1 \text { Normenrest von } K / P \text { mod. jede } p \text { ist. } \\
0, \text { sonst. }
\end{array}\right.
\end{aligned}
$$

Dann ist nach Satz 4

$$
\begin{gathered}
\left(\mathfrak{G}_{0}: 1\right)=2^{\ell \lambda(1-\kappa)} \cdot \frac{\prod_{p} e(p)}{2^{1-\kappa} Q}, \\
\left(\mathfrak{S}_{2}: \mathfrak{S}_{0}\right)=2^{-2-\ell(1-\lambda)} \cdot \prod_{p} e(p) .
\end{gathered}
$$

Also ist

$$
\begin{aligned}
\frac{\left(\mathscr{S}_{0}: \mathscr{H}_{0}\right)}{\left(\mathfrak{W}_{0}: 1\right)} & =2^{-2-\ell(1-\lambda)-\ell \lambda(1-\kappa)+1-\kappa} Q \\
& =2^{-1-\ell+\kappa(\iota \lambda-1)} Q=2^{-1-\iota} Q,
\end{aligned}
$$


denn $\kappa=1$ liefert $: \lambda=1$. Damit ist der Satz bewiesen.

Anschliessend beweisen wir noch den folgenden

Satz 6. Es sei $K$ ein bizyklischer biquadratischer Zahlkörper mit den drei quadratischen Teilkörpern $k_{1}, k_{2}$ und $k_{3}$, und es sei $a_{i}$ ein Ideal von $k_{i}$. Man setze ferner voraus, dass $a_{1} a_{2} a_{3}$ ein Hauptideal von $K$ ist. Dann hat $a_{1} a_{2} a_{3}$ dann und nur dann eine Darstellung $a_{1} a_{2} a_{3}=\left(\alpha_{1}\right)\left(\alpha_{2}\right)\left(\alpha_{3}\right)\left(\alpha_{i} \in k_{i}\right)$, wenn $a_{1} a_{2} a_{3}$ durch eine Zahl $\alpha \in K$ mit $N_{K} \alpha>0$ erzeugt wird. Damit es ein Ideal $a_{1} a_{2} a_{3}$ ohne fragliche Darstellung gibt, ist notwendig und hinreichend, dass zwar -1 Normenrest von $K / P$ mod. jede rationale Primzahl $p$ ist, aber es keine Einheit $\eta$ mit $N_{K} \gamma_{l}=-1$ von $K$ gibt.

Beweis. Ist $a_{1} a_{2} a_{3}=\left(\alpha_{1}\right)\left(\alpha_{2}\right)\left(\alpha_{3}\right)=\left(\alpha_{1} \alpha_{2} \alpha_{3}\right)$, so gilt $N_{K} \alpha>0$ für $\alpha=\alpha_{1} \alpha_{2} \alpha_{3}$. Ist umgekehrt $a_{1} a_{2} a_{3}=(\alpha), N_{K} \alpha>0$, so ist natürlich $N_{K} \alpha=a^{2}(a \in P)$, also ist $N_{k_{i}}\left(\frac{N_{K i k i} \alpha}{a}\right)=1 \quad\left(i=1,2\right.$ und 3). Daher ist nach Hilbert $\frac{N_{K / k_{i}} \alpha}{a}=\alpha_{i}^{\prime 1-s_{i}}$ $\left(\alpha_{i}^{\prime} \in k\right)$ und

$$
\begin{aligned}
\alpha^{2} & =N_{K / k_{1}} \alpha \cdot N_{K / k_{2}} \alpha \cdot\left(N_{K / k_{3}} \alpha\right)^{-s_{3}}=\alpha_{1}^{\prime 1-s_{1}} \alpha_{2}^{\prime 1-s_{2}} \alpha_{3}^{\prime 1-s_{3}} a \\
& =\alpha_{1}^{\prime 2} \alpha_{2}^{\prime 2} \alpha_{3}^{\prime 2} \frac{a}{N_{k_{1}} \alpha_{1}^{\prime} \cdot N_{k_{2}} \alpha_{2}^{\prime} \cdot N_{k_{3}} \alpha_{3}^{\prime}} \cdot
\end{aligned}
$$

Da dann $\frac{a}{N_{k_{1}} \alpha_{1}^{\prime} \cdot N_{k_{2}} \alpha_{2}^{\prime} \cdot N_{k_{3}} \alpha_{3}^{\prime}}$ in $K$ eine Quadratzahl ist, hat $\alpha$ sicher eine Darstellung $\alpha=\alpha_{1} \alpha_{2} \alpha_{3}\left(\alpha_{i} \in k_{i}\right){ }^{11)} \quad$ Damit ist die erste Behauptung bewiesen. ${ }^{12)}$ Die zweite Behauptung folgt ohne weiteres aus der ersten Behauptung und Hilfssatz 14 .

\section{§5. Zusätze und Beispiele.}

Zusatz 1. Es sei $K$ reell und es sei $\eta$ eine Einheit mit $\eta=\eta_{1} \eta_{2} \eta_{3}$ von $K$, wo $\eta_{i} \in \mathbf{e}_{i}, \eta_{i} \geq 1$ und $N_{k_{1}} \eta_{1}=N_{k_{2}} \eta_{2}=N_{k_{3}} \eta_{3}=g(g= \pm 1)$ ist. Setzt man dann

11) Vgl. den Beweis von Hilfssatz 5.

12) Diese Tatsache ergibt sich auch wie folgendes. Es bedeute $\mathfrak{r}_{i}$ ein ambiges Ideai von $k_{i}$. Ist dann erstens $\kappa(K)=0$ und $\mathfrak{r}_{1} \mathfrak{r}_{2} \mathfrak{r}_{3}=(\rho)(p \in K)$, so ist nach Hilfssatz 2 und Hilfssatz $6(\rho)=\left(\rho_{1}\right)\left(\rho_{2}\right)\left(\rho_{3}\right) \quad\left(f_{i} \in k_{i}\right)$. Nach Hilfssatz 15 ist daher notwendig $N_{K} x<0$, wenn $\mathfrak{a}_{1} \mathfrak{a}_{2} \mathfrak{a}_{3}=(x)$ ist und keinesweges $(\alpha)=\left(x_{1}\right)\left(\alpha_{2}\right)\left(x_{3}\right)\left(x_{i} \in k_{i}\right)$ gilt. Ist zweitens $k(K)=1$, $\mathfrak{r}_{1} \mathfrak{r}_{2} \mathfrak{r}_{3}=(\rho)$ und nie $(\rho)=\left(\rho_{1}\right)\left(\rho_{2}\right)\left(\mu_{3}\right)$, so gilt nach Hilfssatz 2 und Hilfssatz $6 r^{2} / r=\varepsilon_{1} \varepsilon_{2} \varepsilon_{3} \eta^{2}$ $(r \in P, \eta$ Einheit von $K)$, und $r$ ist in $K$ keine Quadratzahl. Also ist nach Hilfssatz 3 $N_{K / k} \rho<0$. 


$$
\begin{gathered}
\xi_{0}=\eta_{1} \eta_{2} \eta_{3}+\eta_{1}+\eta_{2}+g \eta_{3} \\
\xi_{1}=\eta_{1} \eta_{2} \eta_{3}+\eta_{1}-\eta_{2}-g \eta_{3} \\
\xi_{2}=\eta_{1} \eta_{2} \eta_{3}-\eta_{1}+\eta_{2}-g \eta_{3} \\
\xi_{3}=\eta_{1} \eta_{2} \eta_{3}-\eta_{1}-\eta_{2}+g \eta_{3} \\
S_{K} \xi_{j}=c_{j} \quad(j=0,1,2 \text { und } 3),
\end{gathered}
$$

so ist

$$
\sqrt{\eta}=\frac{1}{4}\left(\sqrt{c_{0}}+\sqrt{c_{1}}+\sqrt{c_{2}}+\sqrt{c_{3}}\right)
$$

$\ddot{U}$ berdies gehört $\sqrt{\eta}$ dann und nur dann $z u K$, wenn irgendein $c_{j}(j=0,1,2$ oder 3 ) in $K$ eine Quadratzahl ist.

Beweis. Leicht $\mathrm{zu}$ sehen ist zunächst

$$
\sqrt{\eta}=\frac{1}{4}\left(\frac{\xi_{0}}{\sqrt{\eta}}+\frac{\xi_{1}}{\sqrt{\eta_{1}}}+\frac{\hat{\xi}_{2}}{\sqrt{\eta}}+\frac{\xi_{3}}{\sqrt{\eta}}\right)
$$

und

$$
\frac{\xi_{j}^{2}}{\eta}=c_{j} \quad \text { für jedes } j .
$$

Im Falle von $g=1$ ist es klar, dass $\hat{\xi} j \geqq 0$ für jedes $j$ ist. Dies ergibt sich auch im Falle von $g=-1$ in der Tat daraus, dass für jede Einheit $\eta_{i}>1$ von $k_{i} \neq P(\sqrt{5})$ sogar $\eta_{i}>2$ und für jede Einheit $\eta_{i}>1$ von $k_{i}=P(\sqrt{5})$ sogar $\eta_{i}>\frac{3}{2}$ gilt. Damit ist die erste Behauptung bewiesen. Ist nun $\sqrt{\eta} \in K$, so gehört jede $\sqrt{c_{j}}$ ersichtlich zu $K$. Ist umgekehrt $\sqrt{\eta} \notin K$, ist nämlich $K^{\prime}=K(\sqrt{\eta})$ $\neq K$, so ist $S_{K^{\prime} / K} \sqrt{n}=0$. Daher ist für jedes $j$ notwendig $\sqrt{\overline{c_{j}}} \notin K$, was die zweite Behauptung ergibt.

Aus Zusatz 1 folgt ohne weiteres der

Zusatz 2. Es sei $\eta$ eine Einheit mit $N_{k} \eta=1$ eines quadratischen Zahlkörpers $k$. Dann ist ${ }^{13)}$

$$
\sqrt{\eta}=\frac{\sqrt{S_{k}(\eta+1)}+\sqrt{S_{k}(\eta-1)}}{2}
$$

Mittels der bisher erhaltenen Resultate und Zusätze können wir-die Klassenzahl und ein System der Grundeinheiten eines bizyklischen biquadratischen

13) Vgl. [7], S. 395. Hierbei ist ja $S_{k}(\eta+1)=N_{k}(\eta+1), S_{k}(\eta-1)=-N_{k}(\eta-1)$. 
Zahlkörpers $K$ sehr leicht numerisch aus denen der quadratischen Teilkörper von $K$ berechnen.

Beispiel 1. $K=P(\sqrt{ } 6, \sqrt{ } 7)$

$$
\begin{aligned}
& \Delta_{1}=6, \quad \Delta_{2}=7, \quad \Delta_{3}=42 \\
& h_{1}=1, \quad h_{2}=1, \quad h_{3}=2 \\
& \varepsilon_{1}=5+2 \sqrt{ } 6, \quad \varepsilon_{2}=8+3 \sqrt{ } 7, \quad \varepsilon_{3}=13+2 \sqrt{42}, \quad \kappa(K)=0 \\
& \delta_{1}=3, \quad \delta_{2}=2, \quad \delta_{3}=7
\end{aligned}
$$

Beziehungen $z$ wischen $\delta_{i}: \delta_{1} \delta_{2}=1(K), \delta_{3}=1(K)$

Grundeinheiten von $K: \sqrt{\varepsilon_{1} \varepsilon_{2}}, \sqrt{\varepsilon_{3}}, \varepsilon_{2}, Q=4$

Kassenzahl von $K: h=\frac{1}{4} \cdot 4 \cdot 1 \cdot 1 \cdot 2=2$

Nach Zusatz 2 ist

$$
\sqrt{\varepsilon_{1}}=\sqrt{3}+\sqrt{2}, \quad \sqrt{\varepsilon_{2}}=\frac{3 \sqrt{2}+\sqrt{14}}{2}, \quad \sqrt{\varepsilon_{3}}=\sqrt{ } 7+\sqrt{6},
$$

also ist

$$
\sqrt{\varepsilon_{1} \varepsilon_{2}}=\frac{6+3 \sqrt{ } 6+2 \sqrt{ } 7+\sqrt{42}}{2} .
$$

Beispiel 2. $K=P(\sqrt{5}, \sqrt{17})$

$$
\begin{array}{ccc}
\Delta_{1}=5, & \Delta_{2}=17, & \Delta_{3}=85 \\
h_{1}=1, & h_{2}=1, & h_{3}=2 \\
\varepsilon_{1}=\frac{1+\sqrt{5},}{2}, & \varepsilon_{2}=4+\sqrt{17}, \quad \varepsilon_{3}=9+\sqrt{85}, \quad \kappa(K)=1 \\
2 & \\
c_{0}=S_{K}\left(\varepsilon_{1} \varepsilon_{2} \varepsilon_{3}+\varepsilon_{1}+\varepsilon_{2}-\varepsilon_{3}\right)=121 \\
c_{1}=S_{K}\left(\varepsilon_{1} \varepsilon_{1} \varepsilon_{3}+\varepsilon_{1}-\varepsilon_{2}+\varepsilon_{3}\right)=125 \\
c_{2}=S_{K}\left(\varepsilon_{1} \varepsilon_{2} \varepsilon_{3}-\varepsilon_{1}+\varepsilon_{2}+\varepsilon_{3}\right)=153 \\
c_{3}=S_{K}\left(\varepsilon_{1} \varepsilon_{2} \varepsilon_{3}-\varepsilon_{1}-\varepsilon_{2}-\varepsilon_{3}\right)=85
\end{array}
$$

$\mathrm{Da} \sqrt{c_{0}} \in K$ ist, gehört $\sqrt{\varepsilon_{1} \varepsilon_{2} \varepsilon_{3}}$ nach Zusatz $1 \mathrm{zu} K^{14)}$

Grundeinheiten von $K: \sqrt{\varepsilon_{1} \varepsilon_{2} \varepsilon_{3}}, \varepsilon_{2}, \varepsilon_{i, 3}, Q=2$

Klassenzahl von $K: \quad h=\frac{1}{4} \cdot 2 \cdot 1 \cdot 1 \cdot 2=1$

1.1) Vgl. den Beweis von Satz 1. Daraus folgt auch $\sqrt{ } \varepsilon_{1} \varepsilon_{2} \varepsilon_{3} \in K$. 
Ferner ist

$$
\sqrt{\varepsilon_{1} \varepsilon_{2} \varepsilon_{3}}=\frac{1}{4}\left(\sqrt{c_{0}}+\sqrt{c_{1}}+\sqrt{c_{2}}+\sqrt{c_{3}}\right)=\frac{11+5 \sqrt{5}+3 \sqrt{17}+\sqrt{85}}{4} .
$$

Beispiel 3. $K=K(\sqrt{70}, \sqrt{-14})$

$$
\begin{array}{rlrl}
\Delta_{1} & =70, & \Delta_{2}=-14, & \Delta_{3}=-5 \\
h_{1}=2, & h_{2}=4, & h_{3}=2 \\
\hat{\varepsilon}_{1}=251+30 \sqrt{70}, & \zeta_{2}=-1, & \zeta_{3}=-1 \\
\delta_{1}=14, & \delta_{2}=14, & \delta_{3}=5
\end{array}
$$

Beziehung $z$ wischen $\delta_{i}: \delta_{1} \delta_{2} \overline{\overline{2}} 1(K)$

Grundeinheit von $K: \sqrt{-\varepsilon_{1}}, Q=2$

Klassenzahl von $K: \quad h=\frac{1}{2} \cdot 2 \cdot 2 \cdot 4 \cdot 2=16$

Nach Zusatz 2 ist $\sqrt{\varepsilon_{1}}=3 \sqrt{14}+5 \sqrt{5}$, also ist $\sqrt{-\varepsilon_{1}}=3 \sqrt{-14}+5 \sqrt{-5}$.

Beispiel 4. $K=P(\sqrt{2}, \sqrt{-1})$

$$
\begin{array}{lll}
\Delta_{1}=2, & \Delta_{2}=-1, & \Delta_{3}=-2 \\
h_{1}=1, & h_{2}=1, & h_{3}=1 \\
\varepsilon_{1}=1+\sqrt{2}, & \zeta_{2}=\sqrt{-1,} & \zeta_{3}=-1 \\
\delta_{1}=2, & \delta_{2}=2, & \delta_{3}=2 .
\end{array}
$$

Da $\delta_{1}$ "trivial" ist, ist $\varepsilon_{1}$ eine Grundeinheit von $K . \mathbf{D}\left(\varepsilon_{1}^{*}, \zeta_{2}, \zeta_{3}\right) / \mathbf{D}_{0}\left(\varepsilon_{1}^{*}, \zeta_{2}, \zeta_{3}\right)$ wird durch einzige Beziehung $\delta_{2}=1(K)$ vertreten, und daher $\sqrt[4]{-1} \in K$, $Q=2$.

Klassenzahl von $K: \quad h=\frac{1}{2} \cdot 2 \cdot 1 \cdot 1 \cdot 1=1$.

\section{LITERATURVERZEICHNIS}

[1] R. Brauer, Beziehungen zwischen Klassenzahlen von Teilkörpern eines galoisschen Körpers. Math. Nachr., 4 (1951), S. 158-174.

[2] C. Chevalley, Sur la théorie du corps de classes dans les corps finis et les corps locaux. J. Fac. Sci. Imp. Univ. Tokyo, Sec. I, vol. II, Part 9(1933), S. 365-476.

[3] H. Hasse, Zur Geschlechtertheorie in quadratischen Zahlkörpern. J. Math. Soc. Japan, 3 (1951), S. 45-51.

[4] H. Hasse, Über die Klassenzahl abelscher Zahlkörper. Akademie-Verlag, Berlin (1952).

[5] G. Herglotz, Über einen Dirichletschen Satz. Math. Zeitschr., 12 (1922), S. 225-261.

[6] T. Kubota, Über die Beziehung der Klassenzahlẹn der Unterkörper des bizyklischen biquadratischen Zahlkörpers. Nagoya Math. J., 6 (1953), S. 119-127. 
[7] S. Kuroda, Über den Dirichletschen Körper. J. Fac. Sci. Imp. Univ. Tokyo, Sec. I, Vol. IV, Part 5 (1943), S. 383-406.

[8] S. Kuroda, Über die Klassenzahlen algebraischer Zahlkörper. Nagoya Math. J., 1. (1950), S. $1-10$.

[9] H. Nehrkorn, Über absolute Idealklassengruppe und Einheiten in algebraischen Zahlkörpern. Abh. Math. Sem. Hamburg, 9 (1933), S 318-334.

Mathematisches Institut

Universität zu Nagoya 